ISSN: $1130-3743$

\title{
EL CONOCIMIENTO CIENTÍFICO Y SUS CARCOMAS
}

\section{Scientific knowledge and the cankers that go with it}

\section{Le savoir scientifique et ses vrillettes}

\section{Gloria PÉREZ SERRANO}

Universidad Nacional de Educación a Distancia. Departamento de Teoría de la Educación y Pedagogía Social. Paseo Senda del Rey, 7. 28040 Madrid.

Correo-e: gloriaperez@edu.uned.es

Fecha de recepción: marzo de 2011

Fecha de aceptación definitiva: julio de 2011

Biblid [(1130-3743) 23, 2-2011, 19-43]

\section{RESUMEN}

Este trabajo presenta los desafíos del conocimiento científico, su búsqueda y carcomas. El ser humano se ha preocupado por conocerse a sí mismo e indagar en la realidad con el fin de desvelar sus secretos. La ciencia es el gran esfuerzo de la Humanidad por comprenderse a sí misma. La evolución científico-humana precisa de sujetos con formación teórica y conocimiento de los métodos y técnicas de investigación.

La ciencia goza de prestigio, se la cree capaz de resolver casi todos los problemas que tiene planteados la Humanidad. Se le otorga confianza al considerar que sus afirmaciones están fundamentadas, son fiables y fecundas. ¿Qué ofrece la ciencia que la hace incuestionable? Sin duda, presenta límites y carcomas. Hay diversidad de Ciencias. El conocimiento científico está en estado de revolución continua. El desarrollo científico es un bien para la Humanidad. Deberíamos suscitar esta vocación entre los más jóvenes y despertar su curiosidad intelectual.

Palabras clave: ciencia, conocimiento, obstáculos, desafíos, carcomas. 


\section{SUMMARY}

This paper presents the challenges in, the search for and the cankers of scientific knowledge. Human beings have taken pains to learn about themselves and to investigate reality in order to reveal its secrets. Science is humankind's great effort to understand itself. Human scientific evolution requires individuals who have been educated about the theory of research methods and techniques and gained a thorough knowledge in that area.

Science has prestige. It is believed to be capable of solving almost all the problems humanity has ever encountered. Science is trusted, because scientific assertions are regarded as being well grounded, reliable and fertile. What does science offer that makes it unquestionable? It certainly has limits and cankers of its own. There is a diversity of sciences. Scientific knowledge is in a state of constant revolution. Scientific development is an asset for humankind. We ought to fan a vocation for science in our youngsters and awaken their scientific curiosity.

Key words: science, knowledge, obstacles, challenges, cankers.

\section{SOMMAIRE}

Ce travail présente les défis du savoir scientifique, sa recherche et ses vrillettes. L'homme s'est soucié de se connaître et de faire des recherches dans la réalité afin de découvrir ses secrets. La science est le grand effort de l'Humanité pour se comprendre. L'évolution scientifique-humaine a besoin de gens possédant de la formation théorique et des connaissances concernant les méthodes et les techniques de recherche.

La science a du prestige, on la croit capable de résoudre presque tous les problèmes qui affligent l'Humanité. On lui fait confiance car on estime que ses affirmations sont fondées, fiables et fécondes. Que propose la science qui la rend incontestable? Sans doute, elle présente des limites et des vrillettes. Les Sciences sont diverses. Le savoir scientifique est en état de révolution constante. Le développement scientifique est un bien pour l'Humanité. Nous devrions susciter cette vocation chez les jeunes et éveiller leur curiosité intellectuelle.

Mots clés: science, savoir, obstacles, défis, vrillettes.

\section{INTRODUCCIÓN}

A lo largo de la historia, el ser humano se ha preocupado por conocerse a sí mismo e indagar en la realidad en la que vive, con el fin de desvelar sus secretos.

Los métodos y las técnicas empleados en un primer momento han sido muy rudimentarios. Sin embargo, la inquietud a la que nos hemos referido: conocerse a sí mismo y conocer el contexto en el que se vive, ha propiciado su desarrollo. No en vano, se ha definido a la ciencia como «el gran esfuerzo de la Humanidad por comprenderse a sí misma" (Leahey, 1998, 32). 
La evolución científica y humana precisa de sujetos con una buena formación teórica y un conocimiento del manejo de los métodos y técnicas de investigación. De ahí que a un investigador se le pida que desarrolle unas determinadas actitudes.

Ningún investigador puede afirmar que su búsqueda ha terminado, puesto que no existen límites en el conocer, dado que lo que se va descubriendo son verdades parciales y, en este sentido, debemos señalar que el saber científico debe ser, fundamentalmente, precavido, por lo que debe adoptar un carácter de constante provisionalidad. De ahí que la actitud científica genere un estilo de vida que se mueve en una tensión dialéctica entre ruptura y fidelidad. Ruptura con lo que se considera que no sirve, o se considera erróneo, y fidelidad con lo que se ha ido descubriendo y se considera valioso.

Muchos autores proponen una vuelta a la epistemología, a estudiar las cuestiones básicas referentes al criterio de verdad, de certeza y lógica de justificación del conocimiento. Son cuestiones de hondo calado que hoy tiene planteada la Filosofía de la Ciencia, incluso el estudio de la especificidad de los requisitos que exige el conocer científico, así como las reglas y estrategias que identifican al método en la línea que señalaba Lakatos, para el que la teoría de la ciencia no es sino "el perro guardián de las normas científicas". Estas consideraciones "aparecen como fundamentalmente importantes y están cargadas de consecuencias" (Chalmers, 2003, XX).

La ciencia goza de un prestigio, podríamos decir que incluso de un privilegio especial, debido a la confianza que se le otorga al considerar que sus afirmaciones están fundamentadas y van acompañadas de un alto grado de fiabilidad. La imagen que de la ciencia se tiene hoy, en amplios sectores de la sociedad, es debida a la efectividad de los resultados de la investigación científica. Esto hace que se deposite en ella tal confianza que se la crea capaz de resolver, si no todos, casi todos los problemas que tiene planteados la Humanidad. De hecho,

... la legitimidad social que posee hoy la ciencia proviene en buena medida de la convicción general en la utilidad potencial de todos los conocimientos científicos. Es frecuente que un nuevo resultado en la investigación sea anunciado en los medios de comunicación junto con las promesas de sus aplicaciones futuras. Paradójicamente esta imagen va unida también a un temor creciente a que se superen ciertos límites, sobre todo éticos (investigación armamentística, clonación, eutanasia, etc.) y a que se deteriore irreversiblemente el entorno natural debido a un uso incontrolado de esos resultados de la investigación (Diéguez, 2005, 111).

Desde nuestro punto de vista, la ciencia no posee rasgos especiales que la hagan intrínsecamente superior a otras formas de alcanzar el conocimiento. Esta valoración, extendida en nuestra sociedad, lleva a considerar a la ciencia, en muchos casos, como una religión moderna. Ante esta situación, de la mano de Alan F. Chalmers (2003) nos atrevemos a preguntar: ¿Qué tiene la ciencia de especial para alcanzar tan alta valoración? ¿Qué es el método científico al que se le supone 
la clave del éxito? ¿Es lícito transferir este método a otros campos del conocimiento? En definitiva, ¿qué ofrece la ciencia que la hace incuestionable?

La respuesta, a estos interrogantes concernientes a la especificidad de lo científico, no está exenta de una gran dificultad. Como ha sugerido Laudan (1996, 218 y ss.) la preocupación por la ciencia debe centrarse en averiguar cuándo nos encontramos ante una buena teoría, es decir, que sea fiable, fértil y bien fundada, sea o no científica.

El pensamiento actual, respecto a la tradición, defiende posturas epistemológicas más abiertas y plurales, dado que el conocimiento científico no se construye desde una perspectiva única. "Para confirmar científicamente la verdad, es conveniente verificarla desde varios puntos de vista diferentes" (Bachelar, 1974, 14). La ciencia progresa gracias al ensayo y gracias al error, a las conjeturas e impugnaciones, al estudio y a la investigación. Es una tarea intersubjetiva que reclama el concurso y la colaboración de numerosos investigadores. Proporciona un tipo de conocimiento comunicable y público. En la actualidad, no puede negarse la diversidad de la ciencia. Asistimos al surgir de nuevas disciplinas en campos diferentes.

El término ciencia ya no es entendido como un nombre en "singular". La expresión ciencias naturales, por el contrario, es plural y la imagen platónica de un único tipo de conocimiento formal ha sido remplazada por una imagen de empresas que están siempre en flujo, y cuyos métodos de investigación -como pensaba Aristóteles- se adaptan a la naturaleza del caso. Para Toulmin (1990, 165), "en lugar de ser partes diversas de una sola y comprehensiva ciencia unificada, hoy las ciencias representan, más bien, una confederación de empresas con métodos y patrones de explicación para abordar problemas distintos".

En la actualidad, hemos visto surgir disciplinas nuevas aplicadas al estudio de otros tantos campos diferentes, lo que hace difícil negar el hecho de la diversidad de las ciencias. Esto, y todo lo indicado con anterioridad, nos lleva a reflexionar sobre los problemas que tiene hoy planteados el avance en el conocimiento científico.

Por tanto, el motivo de este trabajo se centra en presentar los desafíos del conocimiento científico, así como las dificultades que nos impiden su desarrollo y que nos hemos atrevido a denominar las carcomas de la ciencia.

\section{2. ¿QUÉ PRECISA EL CONOCIMIENTO CIENTÍFICO?}

El ser humano siempre ha manifestado inquietud por conocer la realidad que observa, manipulándola y modificándola con el fin de comprenderla, al mismo tiempo que la transforma. Se enfrenta al reto de describir y explicar a otros la imagen de todo aquello que se le ofrece como conocimiento. Por lo tanto, el conocimiento que aquí defendemos es ese saber consciente y fundamentado, adquirido por el método científico, al que se le exige -como diría Bunge $(1981,9)$ - que sea 
"racional", "Sistemático", "exacto", "verificable" y "fiable». Este conocimiento llamado científico es el que se corresponde con el término griego episteme y se distingue del conocimiento vulgar porque a éste simplemente se le recuerda y no se somete a crítica. El conocimiento científico se opone a la simple subjetividad, a la intuición o al sentido común.

El conjunto de conocimientos que el sujeto humano obtiene sobre la realidad que pretende conocer a través de la actividad orientada a conseguirlo es lo que denominamos ciencia (scire, que significa saber). No obstante, el título de ciencia no se puede aplicar a cualquier conocimiento, sino únicamente a los saberes que han sido obtenidos, como afirma Popper $(1985,68)$, de modo riguroso y contrastable: "La ciencia debe conseguir estructurar sistemáticamente los conocimientos en función de unos principios generales que sirven de explicación y poseen a aquéllos, dando una coherencia general y claridad inexistente anteriormente».

La ciencia persigue avanzar en el descubrimiento de problemas nuevos, generales o profundos y justificar las respuestas correspondientes. "No pretende ser verdadera, ni por tanto final, incorregible y cierta..."-dirá Bunge (1969, 46)- "lo que afirma la ciencia es...» aquello que la hace:

- Ser «más verdadera que cualquier modelo no científico del mundo.

- Capaz de probar, sometiéndola a contrastación empírica, esa pretensión de verdad.

- Capaz de descubrir sus propias deficiencias.

- Capaz de corregir sus propias deficiencias, o lo que es lo mismo, (re)construir representaciones parciales de la estructura del mundo que sean cada vez más adecuadas".

Conviene recordar aquí que la ciencia es una actividad humana sometida, como muchas otras manifestaciones culturales, a cambios históricos.

Señalamos algunos requisitos para acercarnos al conocimiento científico que nos dará entrada al estudio de los "obstáculos epistemológicos", como los denomina Bachelard (1983).

\subsection{Anclajes teóricos}

Necesitamos todas las formas del conocer que nos lleven al descubrimiento de la verdad, porque todas ellas muestran parte de la verdad y todas tienen sus límites.

Existen diferentes modos de aproximarse al conocimiento de la realidad: la ciencia, la fe, el arte, la experiencia, etc. Los humanos somos conscientes de ello, por lo que no debemos prescindir de ninguno. En esta ocasión tan sólo haremos referencia a una de esas modalidades, la del conocimiento científico.

Existen numerosos estudios que ponen de relieve el amplio consenso entre el profesorado universitario (docente/investigador) sobre la importancia de un conocimiento en profundidad de la materia, tanto en el ámbito de la transmisión 
del saber, como en la investigación de la realidad. Desde esta perspectiva, se considera que la formación del docente y del investigador es un aspecto fundamental para el avance de la ciencia y es imprescindible para la formación de los estudiantes.

¿Qué entendemos por un conocimiento en profundidad? Exige hondura y penetración del pensamiento y de las ideas. Cuatro son los elementos que lo caracterizan: un sujeto que conoce, un objeto para conocer, un pensamiento que es medio con el que opera el sujeto en su actividad captadora del objeto y la imagen mental que representa al objeto, en cuanto tal, por medio del concepto e idea.

La epistemología estudia esta relación entre el sujeto y el objeto y todos los problemas que conlleva.

Este modo de conocer es el resultado de una actitud y un esfuerzo especial del sujeto, que le conduce a la sistematización y comprensión cabal de los objetos que estudia y le otorga una certeza superior en sus fundamentos. Por lo tanto, el conocimiento que se precisa para alcanzar una formación adecuada incluye (Gil Pérez et al., 1991, 3-4):

a) Conocer los problemas que originaron la construcción de los conocimientos científicos, las barreras a superar, la evolución de dichos conocimientos, y cómo se llegaron a articular en las distintas disciplinas.

b) Conocer la metodología empleada, la naturaleza del objeto de estudio de cada ciencia, los criterios de validación y de aceptación de las teorías científicas.

c) Conocer el papel de las interacciones con otros saberes, con su aplicabilidad, con la sociedad a la que sirve y su incidencia en la toma de decisiones, etc.

d) Conocer los desarrollos recientes y sus perspectivas, para que el docente y/o investigador, adquiera una visión abierta, dinámica e innovadora.

e) Conocer las aplicaciones de la parcela del conocimiento en el que se trabaja y de otras materias relacionadas con el mismo, que le permitan abordar problemas de frontera, así como, las interacciones con otros campos del saber y los procesos de unificación científica.

Este conocimiento en profundidad es indispensable, tanto en la formación de investigadores que tienen la función de alumbrar el conocimiento, como en la de los docentes encargados de transmitir dicho conocimiento. Bachelard (1974, 10) ilustra esta formación con una imagen: "HHay tan gran distancia entre el libro impreso y el libro leído, entre el libro leído y el libro comprendido, asimilado, retenido!".

En el plano de la formación individual, este autor señala que la persona científica en su formación ha de transitar por tres estadios.

1. El estadio concreto... El espíritu se recrea con las primeras imágenes del fenómeno y se apoya sobre una literatura filosófica que enaltece la naturaleza..., la unidad del mundo y la diversidad de las cosas. 
2. El estadio concreto-abstracto... Adjunta a la experiencia física esquemas geométricos y se apoya sobre una filosofía de la simplicidad.... Está más seguro de su abstracción cuanto más claramente está representada por una intuición sensible.

3. El estadio abstracto... (Las) informaciones son voluntariamente sustraídas... y... desligadas de la experiencia inmediata... (Bachelar, 1974, 11).

Descritos los estadios por los que el aprendiz-investigador ha de transitar para adquirir el espíritu científico, el autor invita al formador a prestar atención a la dimensión afectiva y al interés vital por la investigación desinteresada. Afecto e interés que el formador debe analizar y captar la fuerza con la que fluyen en el investigador novel y que ha de cuidar que se mantenga a lo largo de toda la búsqueda con "paciencia científica", siempre en búsqueda de un conocimiento científico, objetivo, en contraposición con el supersticioso, intuitivo o mítico. De este modo, se tornará en el novel, "claramente consciente y activo, el placer de la excitación espiritual en el descubrimiento de la verdad".

Nunca como en nuestra época el espíritu científico necesita ser defendido, ser ilustrado... Debe forjar la mente con la verdad. El amor por la ciencia debe ser un dinamismo psíquico autógeno. En el estado de pureza logrado por un psicoanálisis del conocimiento objetivo, la ciencia es la estética de la inteligencia (Bachelard, 1974, 13).

Otro tema importante dentro de la formación teórica hace referencia a la demarcación de la ciencia. ¿Cómo delimitar qué es lo científico y qué no lo es?, ¿cómo delimitar la ciencia de otras formas de conocimiento?, ¿qué distingue la ciencia de lo que no es ciencia?

El problema de demarcación de la ciencia se viene planteando con más insistencia desde la década de 1920 con el Círculo de Viena. El objetivo de estos filósofos de la ciencia: Schilick (1882-1936); Carnap (1891-1970); Wittgenstein (1889-1951), consistía en buscar el rasgo, o rasgos diferenciadores, entre el conocer científico y otras formas del conocer. Así tienen su origen los llamados criterios de demarcación.

Trazar la línea de demarcación entre ambos tipos de conocimiento es una reflexión teórica planteada por Popper en 1967, científico reconocido, quien afirmó que el conocimiento científico es aquel que precisa, como criterio básico, la "refutabilidad" o "testabilidad".

Lo que caracteriza a la ciencia no son sólo los enunciados verdaderos ("verificabilidad/confirmabilidad": propuestos por el Círculo de Viena no como criterio de demarcación, sino como búsqueda de sentido), sino la posibilidad de demostrar que sus afirmaciones son falsas ("falsabilidad", Popper, 1934). Esto quiere decir que la ciencia -y con ella la teoría defendida por un científico- se expone a la posibilidad de que ésta sea discutida críticamente y ha de manifestar la capacidad de resistir a dicha crítica.

En 1968 I. Lakatos introduce un nuevo criterio de demarcación de inspiración popperiana, que denomina "progresividad de los programas de investigación", que 
no se basa en un criterio meramente lógico, sino que este criterio sirve para evaluar los avances o estancamientos de los proyectos de investigación. Según Lakatos, un programa de investigación puede pasar por largas fases degenerativas para volver a tornarse progresivo. "Resulta muy difícil -escribe- decidir cuándo un programa de investigación ha degenerado sin remisión posible, si no se exige que exista progreso en cada paso; o cuándo uno de los programas rivales ha conseguido una ventaja decisiva sobre otro" (Lakatos 1983, 193). Por lo tanto, el criterio de demarcación entre ciencia y no ciencia establece que una teoría es científica si es progresiva empíricamente, es decir, si predice hechos nuevos y explica parte de éstos, además de los ya conocidos. Esto implica, también, que sea progresiva teóricamente.

El progreso de la ciencia es un hecho incuestionable hasta convertirse en un rasgo distintivo de la misma. De hecho, la epistemología dedica su atención al estudio de este tema, y en razón de ello ha de dar cuenta a la comunidad científica.

T. Kuhn ha demostrado su influencia en la filosofía de la ciencia y en otros muchos ámbitos del conocimiento a través de su libro La estructura de las revoluciones científicas, publicado por primera vez en 1962. En esta obra analiza el carácter revolucionario del progreso científico.

La revolución implica el abandono de un paradigma por otro, capaz de resolver algunos problemas que el primero no podía resolver. El nuevo paradigma ha de ser asumido por parte de la comunidad científica. Kuhn rechaza la idea de que el cambio revolucionario conduzca a algún tipo de progreso objetivo, dirigido hacia una meta, pero sostiene que hay un progreso unidireccional e irreversible en la capacidad de los paradigmas para resolver problemas (Kuhn, 1970).

Esta manera de entender la naturaleza de la ciencia presenta, para algunos autores, un carácter más descriptivo que racional. Pretende describir las teorías y la actividad de los científicos, por lo que aporta un valor reducido a la teoría de la ciencia.

Llegados a este punto, los filósofos de la ciencia reconocen tener dificultades en la búsqueda de la caracterización de la ciencia para seleccionar aquello que la distingue de los demás tipos de conocimiento (Chalmers, 2003; Diéguez, 2005) cosa nada extraña, ya que, como señala Sáez (1991, 284), en el principio de la humanidad, todo conocimiento era mítico, luego filosófico y hoy pretende ser científico, un conocimiento además no estable y sometido a revisión constante.

Ante esta situación, no es de extrañar que Feyerabend (1975), otro discípulo de Popper, llegara a negar que existiera ningún tipo de distinción relevante entre ambos campos del saber: lo científico y lo no científico. Por el contrario, llega a afirmar que no existe ningún método general para ampliar o examinar nuestro conocimiento y la única descripción del progreso científico es "anything goes" (todo vale). Feyerabend, que fue considerado en su tiempo como anarquista, a raíz de su obra Tratado contra el método (1975), está lejos de ser un enemigo de la ciencia, por el contrario, lo que pretende es hacerla más flexible, menos dogmática y más humana, al ofrecer un camino intermedio donde cada científico pueda utilizar los 
métodos que en cada momento considere pertinentes. Defiende, por tanto, un pluralismo metodológico.

Hay una cierta corriente de pluralismo metodológico en la filosofía contemporánea de la ciencia que puede retrotraerse hasta Feyerabend, y que manifiesta un escepticismo hacia la supuesta "unidad" de la ciencia. Según esta opinión, no debemos exagerar la uniformidad de las ciencias: ciencias diferentes tienen simultáneamente métodos muy diferentes, y estadios diferentes en la historia de una misma ciencia exhiben también métodos diferentes (Preston, 1997, 180).

Feyerabend defiende que no existe un único método científico, como tampoco existen normas que sean aceptadas por todos los científicos de manera universal y tampoco hay una sola verdad en la ciencia.

La teoría de Feyerabend se sitúa dentro de un entramado ético que le permite el cultivo de la libertad individual e implica una actitud humanitaria, según la cual los sujetos se han de considerar libres y democráticos. Es posible que Feyerabend bebiera de las fuentes de Stuart Mill, autor prolífero del s. XIX, gran defensor de la libertad del individuo. En su obra Sobre la libertad (1859), llega a afirmar:

Que la especie humana no es infalible; que sus verdades no son generalmente más que medias verdades, en la mayor parte de los casos; que la unidad de opinión no es deseable a menos que resulte de la más libre y más completa comparación de opiniones contrarias, y que la diversidad de opiniones no es un mal sino un bien, por lo menos mientras la humanidad no sea capaz de reconocer los diversos aspectos de la verdad, tales son principios que se pueden aplicar a los modos de acción de los hombres en no menor medida que a sus opiniones (Mill, 1972, 83).

Conviene tener presente que, desde la sociología de la ciencia, esta cuestión es puramente convencional, puesto que es la propia comunidad científica la que define, en cada momento, lo que se considera o no científico. El conocimiento se incrementa y progresa a base de reflexionar sobre nuestros errores y aprender sobre los mismos. Esta retroalimentación nos ayuda a corregir lo errado y a demostrar la no falsedad.

La existencia de este problema de demarcación de la ciencia no quiere decir que no podamos tener criterios para deslindar lo que se considera científico. Entre los elementos esenciales del conocer científico señalamos: racionalidad, reflexibilidad, sistematización, contraste con la realidad, enunciados que se modifican y corrigen, obtención del saber de manera metódica y rigurosa, uso de un lenguaje y vocabulario específico que conduzca al rigor conceptual, capacidad de predicción, etc.

\subsection{Conocer los métodos y las técnicas}

A la hora de hablar de ciencia, como resultado de acciones sistemáticas fruto de un plan previamente fijado, es obligado hacer referencia a los procedimientos 
que se utilizan para hacer un mejor uso de las facultades que ponemos en juego, para llegar a conocer la realidad y sus fenómenos.

El método es un elemento esencial en la estructura del trabajo científico. Sin método no se puede realizar eficazmente este trabajo, puesto que es imprescindible para la búsqueda de un resultado que no está determinado.

He nacido, lo confieso, con tal inclinación de espíritu que he puesto siempre el sumo placer del estudio, no en oír las razones de los otros, sino en encontrarlas yo mismo con mi propio trabajo (Descartes. Regla X).

La necesidad de marcarse, intencionalmente, un camino basado en estrategias rigurosamente aplicadas para lograr el efecto conveniente hace patente su necesidad.

El método científico no es infalible, es decir, puede mejorarse y tampoco es autosuficiente, lo cual hace referencia a la necesidad de complementación con otros métodos.

El investigador, para aproximarse a la realidad, con el fin de conocerla, ha de utilizar conscientemente las herramientas de su trabajo metodológico, desplegando cualquier estrategia, método o material que tenga a su alcance. Desde este punto de vista, la combinación de múltiples métodos, materiales empíricos, perspectivas, e incluso observadores, focalizados en un estudio singular, debe entenderse como una estrategia que agrega rigor, amplitud y profundidad al trabajo del investigador (Denzin y Lincoln, 1994).

Esta tendencia ha ido perfilando un estilo de investigación en el que se integran distintos métodos en un mismo diseño. Nos referimos, por ello, a la investigación multimétodo.

Tres son las razones que básicamente pueden llevarnos a la integración de distintos métodos en un mismo diseño de investigación:

1. Contar con dos imágenes distintas de una misma realidad o fenómeno social, objeto de estudio.

2. Compensar las limitaciones de un método con las fortalezas de otro.

3. Reforzar la validez de unos resultados.

Estas razones se hallan en la base de las tres estrategias de integración a las que Bericat $(1998,111)$ denomina estrategias de "complementación", de "combinación" y de "triangulación", respectivamente.

No queremos pasar por alto los problemas de legitimidad científica que plantea la investigación multimétodo. Son muchos los autores que participan en este debate y sostienen la tesis de que las posibilidades de integración sólo pueden ser resueltas en el plano metodológico, siempre y cuando la estructura resultante sea, en sí misma, coherente (Perez Serrano, 2007, 11). 


\subsection{Actitud científica}

La actitud siempre hace referencia a una predisposición que orienta al sujeto de ciencia a detectar problemas, a formular preguntas, e interpelar a la realidad, frente a la cual se detiene con el fin de escudriñarla y desentrañar su naturaleza, funciones, características, buscando respuestas, con el fin de conocerla, sin instalarse en una sola y/o única respuesta.

El sujeto que posee esta actitud avanza en las certezas -búsqueda de la verdad-, pero sabe que el camino discurre en la incertidumbre, dado que la realidad es compleja y multidimensional. "Es necesario -dirá E. Morin (1999, 3)- aprender a navegar en un océano de incertidumbres a través de archipiélagos de certezas". Se trata de preguntarse y realizar el esfuerzo por resolver con rigor las cuestiones planteadas. El científico trabaja más motivado por la incertidumbre que por las certezas.

Debemos tener en cuenta que la verdad es frágil, dada la complejidad de la realidad humana. Nadie puede apropiarse de ella. Lo que en un momento admitimos como verdadero puede cambiarse, ser insuficiente o llegar, incluso, a considerarlo un error. En este sentido, debemos estar abiertos y en constante búsqueda de la verdad.

La actitud científica debe llegar a configurar un estilo de vida. La actividad, de algún modo creativa, se halla vinculada a una trabajo esforzado, regular, tenaz y perseverante. Con palabras de López-Yepes $(1995,6)$ subrayamos que el investigador

... es un explorador que camina siguiendo el rastro, las huellas, los vestigios de otros como él. Cuando termina de seguirlos, inicia desde el nuevo punto de partida un recorrido que ha de llevarle a la verdad buscada con obsesión, la que implica atravesar bosques, caminar bajo cascadas poderosas, cruzar ríos indómitos y afrontar otros peligros, el más grande de todos, el desánimo. Pero cuando llega a la meta, cuando descubra aquello que buscaba, saboreará de tal modo el éxito que querrá repetirlo y... probablemente consagrará toda su vida a la aventura de la investigación científica.

Por su parte, K. Popper (1967) llega a decir: "A mí, buscador de verdad, en permanente combate contra el error, lo que me preocupa es hacer vivir las verdades que hacen vivir". Es necesario, en conclusión, que el investigador no se cierre al conocimiento, ni se considere en posesión de la verdad absoluta. Desde nuestro punto de vista, no existe peor frase para un investigador que decir «la búsqueda ha terminado...".

\subsection{Finalidad o búsqueda de una ciencia con consciencia}

Si tenemos claro el concepto y la finalidad de lo que hacemos, si fundamentamos nuestro trabajo y reflexionamos críticamente sobre el mismo, contribuiremos a 
clarificar el complejo bosque del conocimiento y su desarrollo. No en vano hemos asumido como concepto de ciencia que es el gran esfuerzo de la Humanidad por comprenderse a si misma. La lógica intrínseca de los fenómenos naturales, la racionalidad de los procesos y la maravillosa estructura del ser más íntimo de las cosas dan que pensar a la consciencia humana.

Cuando un científico se pregunta: ¿Cuál es el objetivo de mis investigaciones?, ¿qué sentido tiene lo que realizo? La respuesta a estas cuestiones se puede dar desde dos puntos vista que se hacen complementarios: desde la toma de conciencia de lo que hace y desde el comportamiento ético.

La ciencia con consciencia reclama del científico un estado cognitivo que permite la interactuación, interpretación y asociación con los estímulos externos denominados realidad.

Logrando mantener la conciencia (consciencia) en cada situación, se potencia la adquisición, la representación, la interpretación y la utilización de cualquier información relevante, con el objeto de poner sentido a los eventos que ocurren, pudiéndose anticipar a los acontecimientos futuros, dando la capacidad de poder tomar decisiones inteligentes y mantener el control de lo que se investiga.

El científico formula en términos simples lo que ocurre para poder figurarse lo que debe hacer. Es la capacidad para dar respuesta a preguntas tales como: ¿Qué está ocurriendo? ¿Por qué ocurre? ¿Que ocurrirá a partir de ahora? ¿Qué puedo hacer ahora?

El científico no debe dejar de preguntarse. Estas preguntas van más allá de la ciencia y se relacionan tanto con la dimensión cognitiva de la conciencia como con la dimensión ética, que siempre deben acompañarle.

\section{LAS PLAGAS O CARCOMAS DE LA CIENCIA}

En este punto, se presentan algunas de las dificultades que, al considerarlas de gran importancia, no hemos dudado en denominarlas: plagas o carcomas de la ciencia.

Nos vamos a referir más directamente a las plagas que afectan a las Ciencias Humanas y Sociales (CCHHSs), si bien, muchas de ellas, podrían hacerse extensivas a otros ámbitos del conocimiento.

El desarrollo del conocimiento científico es un medio poderoso de detección de errores y de lucha contra las ilusiones. No obstante, los paradigmas que controlan la ciencia pueden desarrollar ilusiones y ninguna teoría científica está inmunizada para siempre contra el error. Además, el conocimiento científico no puede tratar únicamente los problemas epistemológicos, filosóficos y éticos.

La educación debe entonces dedicarse a la identificación de los orígenes de errores, de ilusiones y de cegueras (Morin, 1999, 6). 
Los avances en el progreso de la ciencia no siempre se han llevado a cabo, a lo largo de la historia humana, de una manera fácil y fluida, por el contrario, se han producido a través de desviaciones que proceden de innovaciones o creaciones internas, o de acontecimientos, o accidentes externos: persecución, indiferencia, falta de financiación, falsas refutaciones, etc. Estos hechos fueron parte del paisaje habitual de los grandes pioneros de la historia de la ciencia, pero, aún hoy, muchos investigadores se ven postergados - cuando no amenazados- a causa de sus estudios y trabajos.

Conocer el progreso científico exige contemplar la dialéctica del ensayo y el error. Tanto desde el punto de vista docente como investigador, el error no se puede considerar siempre como resultado de la ignorancia, la incertidumbre, el azar que las teorías conductistas del aprendizaje nos han transmitido. Por el contrario, puede deberse a otros factores, tales como el efecto del conocimiento previo que posee un sujeto y que tenía su interés, incluso sus logros en una etapa determinada y que se manifiesta inadecuado o erróneo en otra posterior. Los conceptos previos, los modelos implícitos, existentes en el cerebro de un sujeto pueden dificultar e impedir las nuevas adquisiciones en el saber. "Los errores de este tipo no son erráticos e imprevisibles, sino que constituyen obstáculos" (Brousseau, 1983, 166). En este sentido, participamos de las famosas afirmaciones de Einstein cuando señalaba que "iTriste época la nuestra! Es más fácil desintegrar un átomo que un prejuicio" o que "el sentido común es el conjunto de prejuicios acumulados a través de los siglos".

Una de las características de estos obstáculos es su resistencia a ser modificados, y es difícil hacerlos desaparecer aunque hayan sido conscientemente rechazados. Debemos reconocer, y lamentar, que este tipo de obstáculo tiene una larga presencia y un profundo peso en nuestra historia científica.

La razón del porqué de estas situaciones quizás haya que buscarla en que no podemos conocer lo que captamos de la realidad externa a nosotros mismos sin intervención de lo que cada uno de nosotros somos. Ni siquiera podemos formular con precisión los conceptos que tenemos en la mente. Y si esto es así en quien formula un contenido mental, cuánta más dificultad tendrá el que ha de captarlo y convertirlo en conceptos, quien también está influido por su propio ser.

Según Gastón Bachelard «hay que plantear el problema del conocimiento científico en términos de obstáculos" $(1974,15)$ ya que en el acto de conocer aparecen necesariamente los entorpecimientos, causas de inercia, que él denomina "obstáculos epistemológicos". Según este autor, se conoce siempre en contra de un conocimiento anterior. Se tratará entonces de superar obstáculos tales como la opinión, las costumbres intelectuales, el conocimiento empírico inmediato, por ejemplo, en donde lo que cree saberse ofusca lo que debiera saberse. Bachelard nos señala así el lado fecundo del obstáculo, ya que superándolo es como se produce el conocimiento científico.

Una dificultad inminente para realizar muchos de nuestros trabajos e investigaciones es lo que se denomina como aquella desviación o limitación del 
pensamiento científico que tiende a reducir relaciones u objetos nuevos a los ya conocidos. Debemos reconocer y lamentar que este tipo de obstáculo tiene una larga presencia y un profundo peso en la formación superior.

Para poder conocer las condiciones históricas del progreso de la ciencia, hay que plantear el problema no sólo en términos de obstáculos externos, sino «en el acto mismo de conocer íntimamente, donde aparecen, por una especie de necesidad funcional, los entorpecimientos y las confusiones. ... causas de estancamiento y hasta de retroceso, causas de inercia" (Bachelard, 1974, 15).

Es necesario romper estas barreras para poder "conocer en contra de un conocimiento anterior, destruyendo conocimientos mal adquiridos o superando aquello que, en el espíritu mismo, obstaculiza a la espiritualización" (Bachelard, 1974, 15).

El conocimiento científico avanza a través de continuas rupturas epistemológicas. Es decir, de graduales rectificaciones de errores precedentes, superando los esquemas teóricos convencionalmente aceptados. Sin embargo, dichas rupturas no son pasos fáciles de dar, ya que siempre se encuentran con resistencias o reacciones que impiden el avance científico. Esto es lo que Bachelard llama "obstáculos epistemológicos", que son ideas que obstaculizan el surgimiento de otras nuevas: hábitos intelectuales arraigados, teorías científicas que funcionan como dogmas y, sobre todo, dogmas ideológicos que dominan a las diferentes ciencias, además de opiniones altamente aceptadas.

La idea principal de Bachelard es que, en el futuro, el conocimiento se basará en la negación del conocimiento actual. Profundiza en las consecuencias epistemológicas de la que ha sido una mutación fundamental en la ciencia del siglo XX.

En este contexto, Bachelard acuña la noción de "ruptura" epistemológica; los avances en la ciencia no sólo requieren una acumulación, requieren una ruptura con los hábitos mentales del pasado. Los avances se producen, pues, venciendo resistencias y prejuicios, aquellos que pertenecen al cuadro conceptual y a las imágenes dominantes en la configuración epistemológica que ha de superarse.

Para Bachelard $(1973,193)$, es indudable que "El pensamiento científico reposa sobre un pasado reformado. Está esencialmente en estado de revolución continua".

\subsection{Dogmatismo}

Es un modo de funcionamiento cognitivo, social y cultural, totalmente opuesto al modo científico de conocer la realidad. El dogmático sostiene que el conocimiento adquirido, hecho propio y la formulación que hace del mismo son verdades absolutas de manera arrogante y sin posibilidad de réplica. Vive apoyado en sus certezas, es sectario y considera que los que no piensan como él se hallan en el error. Él está, se siente, en la verdad y no tiene por qué preocuparse de verificar su validez, ni de contrastarla con otros. Los conflictos suelen producirse entre personajes fanáticos, como el que se acaba de describir, que se sienten depositarios de una idea vivida como certeza absoluta. 
La producción del conocimiento siempre estará condicionada por la situación de quien, o quienes, lo producen. En la ciencia no hay verdad cerrada y concluida $\mathrm{y}$, como hemos señalado en otras ocasiones, siempre puede revisarse, por ello debe ser antidogmática. La actitud de búsqueda y duda es permanente. Precisamente, la fortaleza de la ciencia está en considerar que las verdades son parciales y siempre sujetas a corrección. La persona que piensa con actitud científica comprende la realidad en la dialéctica de lo objetivo y lo subjetivo. El desafío del pensar no se obtiene mediante una técnica, receta o método.

El dogmatismo constituye en sí mismo una de las principales carcomas de la ciencia, puesto que, como se ha indicado anteriormente, se apoya en certezas absolutas y se cierra a nuevas búsquedas, suele usar argumentos de autoridad como criterio de verdad. El dogmatismo interiorizado pone anteojeras, lo que conduce a una visión de la realidad distorsionada. El dogmático se mira a sí mismo y se considera el ombligo de la ciencia. Esta manera de pensar se puede calificar de provinciana al no considerar los fenómenos desde la multidimensionalidad y complejidad de los mismos.

\subsection{Relativismo}

Es considerado, a menudo, como una actitud, y/o como una teoría filosófica, que niega la validez absoluta y universal de cualquier idea, principio y teoría. El conocimiento humano y lo que se entiende por verdad están en dependencia no sólo del objeto que se pretende conocer, sino también de las circunstancias y las características del sujeto que conoce.

Se confunde, a veces, con el subjetivismo y el escepticismo. En el subjetivismo, hay un predominio de nuestro modo de pensar o sentir sin hacer referencia al objeto; en el escepticismo, va más allá, niega la existencia de la verdad al considerar que el ser humano es incapaz de conocerla.

En un sentido general, ser relativista, en lenguaje vulgar, es encontrar que todo es equivalente y que no vale la pena hacer diferencias. Una persona relativista piensa que, puesto que no es posible conseguir una certeza absoluta, no merece la pena seguir buscando. Por otra parte, es necesario distinguir bien lo que el relativismo es de lo que no es. En este sentido, no es relativismo aceptar que pueden existir diferentes opiniones sobre un hecho o fenómeno, lo que por otra parte es obvio.

Es importante, sin embargo, tener en cuenta que para llevar a cabo una investigación no existen recetas universalmente válidas, menos aun si se trata de una investigación cualitativa. Es preciso seguir un proceso sistemático vertebrado por el objetivo que queremos conseguir. No podemos caer en el relativismo metodológico e, incluso, en el anarquismo de Feyerabend (1981), de "todo vale". No se puede olvidar que todo vale, pero no todo vale lo mismo. Tampoco es recomendable seguir un esquema lineal tipo, estandarizado, simplemente porque haya dado 
resultado en otros estudios. Desde esta óptica nos permitimos hacer una referencia a Gramsci:

\begin{abstract}
Creer que se puede hacer avanzar una investigación científica aplicándole un método tipo, elegido porque ha dado buenos resultados en otra investigación, a la que se adaptará naturalmente, es una extraña alucinación que tiene muy poco que ver con la Ciencia. Existen, sin embargo, criterios generales que puede decirse que constituyen la conciencia crítica de todos los científicos, cualquiera que sea su especialización, y que deben estar siempre presentes espontáneamente en su labor $(1973,29)$.
\end{abstract}

El proceso de investigación no se puede considerar lineal e inflexible en las CCHHSS (Ciencias Humanas y Sociales). No se trata de un conjunto de etapas definidas mecánicamente, como una escalera en la que iniciado el primer paso ya no se puede volver atrás, en donde no se pueden realizar dos o más etapas de forma paralela, si la práctica demuestra que esto es posible. Esta flexibilidad metodológica, necesaria para el conocer científico, nada tiene que ver con el relativismo.

Entre los obstáculos que presenta el relativismo a la ciencia, podemos mencionar: a) el considerar que no existe conocimiento científico verdadero, sino que éste dependa del sujeto que conoce, de la captación sensorial que realiza y del medio físico y sociocultural en el que busca el saber; b) el relativismo metodológico del "todo vale" puede conducir al anarquismo, y c) la indiferencia ante los resultados conseguidos por el saber científico.

El conocimiento científico se opone al relativismo en tanto que es igual para todos los seres humanos, con independencia del lugar de producción o del lenguaje natural en que se comunique.

\title{
3.3. Pragmatismo-eficientismo
}

Esta corriente se caracteriza por insistir en las consecuencias de utilidad, practicidad y eficacia, como componentes esenciales de la verdad científica. El pragmatismo se mide por el éxito que tenga en la práctica. Si algo no persigue un fin o uso determinado, en la solución de problemas, no hay razón para que tal cosa exista. Al pragmatismo no le interesa el conocer por conocer, sino la utilidad y la eficacia. Valora el desarrollo científico-tecnológico-eficientista frente al humanismo.

El eficientismo, por su parte, busca conseguir lo mejor en poco tiempo. Su lema es lograr el máximo rendimiento con el mínimo esfuerzo. Presenta un carácter utilitario y sirve para satisfacer, de manera inmediata, las necesidades de cada persona.

Necesitamos comprender el papel que juega el pragmatismo-eficientismo en nuestra formación académica. En este momento, en la aplicación del diseño del nuevo Espacio Europeo de Educación Superior (EEES), es preciso no descuidar la 
integración de las distintas perspectivas y, por supuesto, no olvidar la humanista, para evitar el sesgo profesionalizante y mercantilista al que se orienta. La visión pragmatista-eficientista sólo, al ser reduccionista, no debe configurar el objetivo final de la formación.

Lo mejor que tenemos son las personas. Todos los logros y recursos materiales que ha venido acumulando la sociedad actual, y con ella la Humanidad, sólo tienen sentido desde el valor primordial y la calidad del ser humano que aprende, enseña, investiga, colabora y contribuye a la evolución permanente de esta sociedad. Las personas son la mejor demostración del vigor, la capacidad y la proyección de futuro de cada generación. El valor de la persona es un hilo que nos conduce a una educación renovada y a unas estructuras científico-tecnológicas más desarrolladas. Toda investigación especialmente en la CCHHSs no debe apartarse de esta consideración.

Estas carcomas, la excesiva búsqueda del pragmatismo-eficientismo -junto con la disolución del valor humano-, abren un espacio de reflexión o de duda, a fin de considerar su presencia (el valor de lo humano) casi desaparecida en esta corriente de pensamiento. No debemos caer en el simple hacer "ingeniera humana-social" que apoya y acrecienta el conductismo, porque inhibe y restringe las posibilidades creadoras del ser humano. Lo más grave es que propicia el relativismo y justifica cualquier cosa, diluye la responsabilidad y nos hace cómplices de la situación.

\subsection{Ciencia-tecnología-industria}

Esta trilogía se convierte en plaga, al ser la protagonista indiscutible que condiciona el conocimiento mismo y su desarrollo. Lo más característico de la sociedad europea del conocimiento -como se refleja en la Declaración de Bolonia (1999) y en sus manifestaciones posteriores- es quizás el hecho de que "la ciencia se ha convertido ella misma en un tipo lucrativo de actividad económica, en forma de I $+\mathrm{D}$, orientada a la producción de conocimientos nuevos, útiles sobre todo, al tejido industrial" (Rioja, 2007, 59). Todavía estamos a tiempo de evitar que el panorama científico español se convierta en un erial. Hay que reconocer que estamos en grave dificultad y que no sabemos cómo vamos a salir de este atolladero europeísta, o tal vez pseudoeuropeísta.

El carácter fuertemente restrictivo de este modo de entender la sociedad del conocimiento se puede perfilar a través de los rasgos siguientes: concepción económica y mercantilista del conocimiento, competitividad e innovación, cambio vertiginoso exigido por la innovación, control del presente por el futuro, etc.

Las carcomas, en este campo, se encuentran en la finalidad marcada por las reglas y valores del mercado. El nuevo paradigma educativo no gira en torno a la creación del conocimiento, sino a la adquisición de competencias, habilidades y destrezas que demanda el mercado de trabajo. ¿Dónde está la prioridad?, ¿en el Homo habilis, "faber", o en el Homo sapiens? 
El tiempo necesario para transformar un conocimiento básico en ciencia aplicada y éste en tecnología se reduce cada vez más [...] La emergencia de un verdadero sistema de ciencia-tecnología-industria [es] cada vez más poderoso y más autónomo frente a otros poderes [...] Con ello, la ciencia se ha transformado en el primer factor de producción [...] (Lamo de Espinosa et al., 1994, 39).

Estamos llegando a la situación de que el conocimiento no se considera como un derecho de los ciudadanos sino como un servicio internacional que puede comprarse, venderse y evaluarse con arreglo a criterios empresariales (Rioja, 2007, 61).

\subsection{La hiperespecialización}

En este punto nos hemos apoyado en la reflexión que hace Edgar Morin (1999, 18-19) y de su mano hacemos nuestras algunas de sus afirmaciones.

La hiperespecialización es aquel tipo de saber que fragmenta, diluye y reduce el conocimiento científico. Lo fragmenta, cuando extrae una parte del todo y lo convierte en objeto de estudio exclusivo y excluyente, aislándolo del conjunto de las partes que componen el todo y del contexto en el que se ubica, quebrantando la relación de la parte con el todo. Diluye, cuando, al parcializar el todo, rompe la conexión entre las partes y lo esencial al todo queda pulverizado entre los fragmentos. Reduce, cuando disminuye el conocimiento de un todo a una de las partes, llegando a restringir lo complejo a lo simple.

La supremacía de un conocimiento dividido en disciplinas hace difícil comprender la relación entre las partes y el todo y viceversa. Es preciso generar un conocimiento utilizando aquellos métodos que permiten aprehender los objetos en su contexto, captando su complejidad y las relaciones existentes entre el todo y las partes.

En esta manera fragmentada, dispersa y desunida de considerar los saberes, se reflejan algunas carcomas que a menudo quebrantan los contextos, las globalidades y las complejidades, impidiendo un conocimiento pertinente.

La primea carcoma que mencionamos es la ruptura entre ciencias humanas y ciencias experimentales, así como la excesiva parcelación disciplinar.

Las realidades globales, complejas, se han quebrantado; lo humano se ha dislocado; su dimensión biológica, incluyendo el cerebro, está encerrada en los departamentos biológicos; su dimensión psíquica, social, religiosa, económica están relegadas y separadas las unas de las otras en los departamentos de ciencias humanas; sus caracteres subjetivos, existenciales, poéticos se encuentran acantonados en los departamentos de literatura y poesía. La filosofía que es, por naturaleza, una reflexión sobre todos los problemas humanos se volvió a su vez un campo encerrado en sí mismo.

Los problemas fundamentales y los problemas globales son evacuados de las ciencias disciplinarias (Morin, 1999, 18). 
Al reducir el conocimiento del todo a uno solo de sus aspectos, estamos perjudicando al conocimiento mismo, porque, por un lado, se pierde de vista su organización global y, por otro, las propiedades nuevas que aparecen cuando las partes están en relación. Este reduccionismo elimina todo lo que no sea cuantificable y medible y suprime así lo humano de lo humano.

Es cierto que la era Da Vinci pasó y ya nos encontramos lejos del saber renacentista, poseedor de todos los saberes existentes en la época. Hace ya siglos que nadie puede decir yo lo sé todo. Una inteligencia parcelada, mecanicista y reduccionista rompe lo complejo del mundo, separa lo que está unido, lo multidimensional queda reducido a la consideración de uno solo de sus aspectos. En estas condiciones, las mentes pierden sus aptitudes naturales de integración y contextualización de los saberes. Esto conduce, en el comportamiento social, al debilitamiento de la responsabilidad de lo comunitario y de la solidaridad: "a mí no me toca", "no es mi problema". Con estas afirmaciones nos situamos lejos del sentir de Unamuno cuando expresa: "El mundo y yo nos hacemos mutuamente".

\subsection{Dificultad de constituir equipos interdisciplinares}

El trabajo científico en CCHHSs, debido a su complejidad específica, requiere, más que otras disciplinas, de los equipos interdisciplinares. Es necesario estudiar el problema desde diferentes perspectivas con objeto de aportar enfoques y orientaciones plurales. La interdisciplinariedad descansa en saber conjugar con arte la aportación de las diferentes representaciones mentales, aquellas que se consideren más adecuadas para vincularlas al proyecto científico, objeto de estudio. El trabajo interdisciplinar ha de dar valor a la aportación específica de cada especialista.

Trabajar siempre sobre lo mismo, del mismo modo y con las personas de la misma especialidad, lleva a veces a esterilizar la producción. Esto sucede en los cultivos. Si se siembra siempre lo mismo y sucesivamente, al cabo de varios años el terreno deja de producir. En la ciencia puede suceder algo similar. Conviene pararse a pensar, cambiar las perspectivas, ampliar horizontes y enriquecer los equipos con personas procedentes de otros campos de conocimiento, sin olvidar los sujetos que, en la práctica, trabajan a pie de obra sobre el tema.

Entre los múltiples retos a conquistar, uno de los que tenemos que afrontar es superar la organización legislativa en áreas de conocimientos y departamentos, que ha reforzado la tendencia a encerrarnos en guetos profesionales y, como consecuencia, en conceptualizaciones y metodologías dominantes. Tendremos que hacer un esfuerzo por escucharnos más, discutir más y estar dispuestos a aprender unos de otros (Deandaluze, 1998). La organización, en áreas de conocimiento, está incidiendo en la división cerrada en compartimentos estancos, que no propicia la relación ni la convivencia con áreas afines. De este modo, poco a poco, se va perdiendo la visión global y cerrando posibilidades que inciden, sin lugar a dudas, en la creación de grupos de investigación más plurales. 
Es necesario debatir y contrastar ideas con personas procedentes de otros campos del saber. Uno de los riesgos más frecuentes del trabajo en equipo se cifra en que aparezcan actitudes que dificulten la apertura a ideas nuevas, o que quieran hacer prevalecer las propias (dogmatismo). Ya Habermas $(1987,41)$ indicaba que «... quien sistemáticamente se engaña a sí mismo se está comportando irracionalmente, pero quien es capaz de dejarse ilustrar sobre su irracionalidad, dispone de la racionalidad del otro, que tiene la fuerza de comportarse reflexivamente frente a su propia subjetividad, y penetrar en sus conexiones irracionales».

Los equipos, o grupos, interdisciplinares no están exentos de los problemas que surgen al integrar personas muy preparadas frente a las menos preparadas en su ámbito de conocimiento, que deben estar dispuestas, unas y otras, a hacer una serie de concesiones, en orden a lograr una buena dinámica de funcionamiento. Es necesario coordinar la formación intelectual y los intereses individuales, así como las posturas ideológicas de sus miembros. Estos elementos modelarán, en gran medida, el desarrollo y las metas del equipo.

Hacemos referencia a algunas dificultades y barreras, con las que puede encontrarse un equipo, por su especial incidencia en la creación de grupos de estudio e investigación:

- Mayor valoración teórica que práctica del trabajo en equipo

En las CCHHSS existen visiones diversas respecto de este tema. Ha habido cambios importantes sobre lo que representa la teoría para la práctica y en general sobre la relación teorico-práctica.

Las posiciones mayoritariamente asumidas hoy sostienen que la relación entre teoría y práctica no podemos entenderla como una dependencia de la práctica respecto de la teoría. Tampoco podemos reducir el concepto de teoría al de conocimiento científico, ni a un solo tipo de conocimiento, ni a una única teoría (Clemente Linuesa, 2007, 26).

En general, el trabajo en equipo tiende a valorarse desde una perspectiva teórica, si bien se encuentra con grandes dificultades en la práctica. Estudios realizados sobre el tema señalan como dificultades:

- Mentalidades diferentes.

- Tradición de formación individualista.

- Miedo a perder la autonomía, temor o inseguridad.

- Es un proceso lento.

- Tiempo para reunirse.

- Conjugar las tareas de estudio, investigación, docencia y gestión

Se percibe una necesidad creciente de realizar investigaciones en grupo. No obstante, este modo de trabajo se hace difícil en la dinámica de la vida cotidiana, 
donde es preciso atender a diversas actividades docentes y administrativas y/o de gestión que no admiten demora y ocupan gran parte de nuestro tiempo.

- $\quad$ Presión de publicaciones

Nos hallamos sometidos a una productividad cada vez más acusada en determinados ámbitos. Puede llevar a la búsqueda de la producción individual y sesgar el trabajo en equipo que tanta riqueza puede aportar a la mejora de la sociedad, si bien, hasta ahora, se hallaba menos valorado en los baremos oficiales.

- Dotación de recursos económicos

El investigador se halla sometido, también, a presiones de índole económica. Los recursos con los que cuenta este tipo de investigación son mínimos, comparándolos con los que se conceden a los de carácter tecnológico o biomédico. Ello conduce a que los equipos se vean obligados a funcionar con presupuestos exiguos.

- División en la comunidad científica

A diferencia de otros campos científicos que dan la impresión de gozar de una envidiable unidad, la investigación en CCHHSS, en ocasiones, se caracteriza por la división y la rivalidad. Dicha división podría ser debida a los constantes cambios y reorganizaciones, la carencia de consenso y de inseguridad.

Conviene subrayar la falta de consenso de la comunidad científica en muchos aspectos relacionados con la investigación (prioridades, metas, resultados, equipos, horizontes, etc.), se percibe falta de claridad en los criterios de evaluación y de selección de proyectos. Criterios, a veces, desconocidos por los investigadores, lo que genera cierta inseguridad y a veces desánimo en la comunidad científica. ¿Para qué esforzarse? ¿Qué horizontes se vislumbran?

- $\quad$ Definición de prioridades

El partido político de turno, en el Gobierno del Estado y/o Comunidad Autónoma, tiende a dejar su impronta en todos los ámbitos y también en los que considera prioritarios, como son la Ciencia y la Universidad, lo que lleva a muchos equipos a modificar, redefinir e incluso cambiar su línea de investigación por unos años, si quieren seguir subsistiendo como tal. Esto obliga a reformular planteamientos y cambiar de horizonte temático, lo que incide en la calidad de la investigación y sobre todo en la posibilidad de contar con líneas de trabajo consistentes.

Los grupos, al ser interdisciplinares, funcionan como un catalizador que induce, propicia, acelera y transforma el conocimiento y también tienen capacidad para eliminar los elementos contaminantes. Por lo tanto, en el funcionamiento de 
los equipos o grupos interdisciplinares es donde se reflejan con más claridad las carcomas, plagas y dificultades que han sido presentadas en este trabajo.

Conviene tener presente que en el momento actual la tendencia en investigación no sólo consiste en funcionar como grupos interdisciplinares, sino en constituir redes de grupos entre diferentes universidades que investigan sobre un mismo tópico, o tema, con el fin de profundizar en el mismo desde diferentes ópticas y perspectivas. La tendencia actual acusa preferencia por la internacionalización de la investigación. En momentos de crisis económica es muy importante aprovechar los escasos recursos y fomentar redes de grupos de investigación, nacionales e internacionales.

El objetivo que persigue esta modalidad de investigación es (Resolución, n. ${ }^{\circ}$ 12174. BOE, n. ${ }^{\circ} 168(14 / 7 / 2011)$ :

... favorecer la participación española en convocatorias bilaterales o multilaterales para realizar proyectos de investigación conjuntos de dimensión internacional y multidisciplinar, cuya interacción aporte un valor añadido significativo, entre distintos países que se consideren estratégicos en el Programa de Internacionalización de la I+D (art. 34. ${ }^{\circ}$.

Asimismo, se pretende satisfacer las necesidades de personal altamente cualificado de las instalaciones científicas nacionales o empresas españolas, y favorecer, en su caso, la presencia de especialistas españoles en organismos internacionales de investigación de excelencia (art. 2).

\section{A MODO DE CONCLUSIÓN}

Finalmente queremos manifestar que:

- Todo descubrimiento hecho o realizado según las reglas de la investigación científica es un bien para el ser humano, con independencia de la aplicación concreta que tenga.

El desarrollo científico es un bien para la Humanidad, por lo que deberíamos ser capaces de suscitar esta vocación entre los más jóvenes y despertar su curiosidad intelectual. Se trata de fomentar un pensamiento de calidad que es concebido como pensamiento racional, reflexivo y propositivo, que decide qué hacer y qué creer, a la vez que es capaz de reconocer y analizar los argumentos propios y ajenos.

- El conocimiento científico debe ser difundido, hacerlo salir de sus fronteras habituales, exclusivas y excluyentes y promover la curiosidad, a la vez que enseña a la sociedad a dudar de sus postulados.

Aunque suele afirmarse que el estudiante es el objetivo-diana sobre el que pivota la institución de Educación Superior, en la práctica no se tiene en cuenta en los programas la necesidad de desarrollar el pensamiento crítico: racional, reflexivo y propositivo de los alumnos. Se hacen esfuerzos parciales, pero no se define e integra como plan estratégico de aprendizaje. 
- La rigidez en la transmisión de la ciencia se aprecia en la postura omnipotente que concibe a la ciencia como capaz de explicar, de una vez para siempre, todo fenómeno observable y se la llega a considerar como verdad absoluta, sin dejar espacio para el desarrollo del cuestionamiento, aspecto que caracteriza una mente abierta. Se considera a los conocimientos propios como verdades incontrovertibles. Se "dice" como si se estuviera afirmando una verdad definitiva y evidente, por lo que escapa a cualquier discusión y se mantiene con falsa seguridad.

La educación que es a la vez transmisión de lo viejo y apertura de la mente para acoger lo nuevo se halla en el corazón de esta nueva visión. Este desarrollo científico debe operar, siempre y en cualquier circunstancia, en el marco de los principios éticos.

- La alianza entre ciencia y consciencia pasa necesariamente por el reconocimiento del principio fundamental: el respeto de la dignidad humana. Es el principio sobre el que se basan nuestras sociedades democráticas que todo ser humano posee una dignidad inalienable, que le es connatural, no concedida por autoridad alguna, independiente de su religión, clase social, edad, sexo o cultura.

- Nos preguntamos: ¿Cómo podemos pulverizar las carcomas de la ciencia? Creemos que el punto fuerte está en la formación adecuada y pertinente de investigadores. Se precisan científicos comprometidos con la problemática de su sociedad y de su tiempo. Coincidimos con Ciriaca Morano en considerar como...

imprescindible evidenciar ante los órganos de poder que, desde hace años, estamos soportando en nuestro país un proyecto educativo que colabora a formar individuos sin consciencia de sus propias raíces culturales, con una seria crisis del sentido moral, sin posibilidades de desarrollar un juicio crítico basado en un razonamiento sistemático y con un uso del lenguaje cada vez más pobre y menos preciso. Hasta qué punto todos estos datos tienen o no que ver con la progresiva disminución de las disciplinas de humanidades en el aula, sería discutible, pero quienes estamos convencidos de que hay una relación de causa-efecto, encontramos razones suficientes para luchar por potenciarlas $(2007,5)$.

- Es cierto que el desarrollo de la ciencia avanza paralelamente con el desarrollo tecnológico, pero debería extenderse a todos los campos posibles de la sociedad. La ciencia no es una actividad desgajada del resto de la vida del ser humano y, como tal, debe estar limitada por la estricta observancia de la conciencia ética.

Como humanistas, concluimos que podemos "complacernos y admirarnos del alto nivel de desarrollo tecnológico al que la sociedad está llegando, pero, también como humanistas, podemos tener la lucidez de intuir que el desarrollo en civilización no se identifica siempre y necesariamente con el de humanización" (Morano, 2007, 7). 
GLORIA PÉREZ SERRANO

EL CONOCIMIENTO CIENTÍFICO Y SUS CARCOMAS

\section{REFERENCIAS BIBLIOGRÁFICAS}

BACHELARD, G. (1973) El materialismo racional. Barcelona, Paidós.

- (1974) La formación del espíritu científico (3. ${ }^{a}$ ed.). Madrid, Alianza.

- (1978) La dialéctica de la duración. Madrid, Villalar.

BERICAT, E. (1998) La integración de los métodos cuantitativos y cualitativos. Barcelona, Ariel.

Brousseau, G. (1983) Les obstacles épistèmologiques et les problémes en didactique en mathématiques. Recherches en Didactique des Mathématiques, vol. 4 (2), 165-198.

Bunge, M. (1969) La investigación científica. Barcelona, Ariel.

- (1981) La ciencia, su método y su filosofía. Buenos Aires, S. XX.

CHAlmers, A. F. (2003) ¿Qué es esa cosa llamada ciencia? Madrid, Siglo XXI.

Clemente Linuesa, M. (2007) La complejidad de las relaciones teoría-práctica en educación. Teoría de la Educación. Revista Interuniversitaria, 19, 25-46.

DEANDAluZE, I. (1998) Algunos retos metodológicos. Revista de Investigación Educativa, 19 (1), 7-24.

DENZIN, N. K. y LINCOLn, Y. S. (eds.) (1994) Handbook of qualitative research. Thousand Oaks (California), Sage.

DescarTes, R. (1637) Le Discours de la Méthode cartésienne. Leiden (Países Bajos), Ian Maire.

- (2003) Discurso del método. Madrid: Tecnos.

DiéGuez, A. (2005) Filosofía de la Ciencia. Madrid, Biblioteca Nueva.

FEYERABEND, P. (1975) Against Method: Ontline or fan anarchistic theory of kwowledge. London, New Left. (Versión castellana: Tratado contra el método. Madrid, Tecnos).

- (1981) Contra el método. (Trad.: F. Hernán). Barcelona, Ariel.

Gil Pérez, G.; Beléndez Vázquez, A.; Martín García, A. y Martínez Torregrosa, J. (1991) La formación del profesorado universitario de materias científicas: contra algunas ideas y comportamientos de "sentido común". Rev. Interuniversitaria de Formación del Profesorado, n. ${ }^{\circ}$ 12, sep.-dic., 43-48.

GÓMEZ GarCía, P. (coord.) (2000) Las ilusiones de la identidad. Valencia, Frónesis.

GramsCI, A. (1973) La política y el Estado moderno. (Trad.: Jordi Solé-Tura) (2. ${ }^{a}$ ed.). Barcelona, Ed. Península.

Habermas, J. (1987) La Acción Comunicativa. Tomo I. Madrid, Taurus.

KUHN, T. S. (1971) La estructura de las revoluciones cientificas. Madrid, FCE.

LAKATOS, I. (1983) La metodología de los programas de investigación científica. Madrid, Alianza.

LAMO DE ESPINOSA, E. et al. (1994) La sociología del conocimiento y de la ciencia. Madrid, Alianza Editorial.

LAUDAN, L. (1986) El progreso y sus problemas. Madrid, Ediciones Encuentro.

- (1996) Beyond Positivism and Relativism. Oxford, Westview Pres.

LEAHEY, T. H. (1998) Historia de la Psicología. Madrid, Prentice Hall Iberia.

LÓPEZ-YEPES, J. (1995) La aventura de la investigación científica. Guía del investigador y del director de investigación. Madrid, Síntesis.

MiLL, J. S. (1859/1972) Sobre la libertad. Madrid, Aguilar.

Morano, C. (2007) Reflexiones sobre la "defensa" de las Humanidades. CSIC, Documento Policopiado.

MORIN, E. (1999) Los siete saberes. Paris, UNESCO. 
PÉrez SERrano, G. (2007) Desafíos de la Investigación Cualitativa. Santiago de Chile, Centro de Formación de Profesores.

POPPER, K. (1967) El desarrollo del conocimiento científico: conjeturas y refutaciones. Buenos Aires, Paidós.

- (1973) La lógica de la investigación científica. Madrid, Tecnos.

- (1979) El desarrollo del conocimiento científico: conjeturas y refutaciones. Barcelona, CEAC.

- (1985) La lógica de la investigación científica. Madrid, Tecnos.

Preston, J. (1997) Feyerabend: Philosophy, Science and Society. Cambridge, Polity Press.

RiojA, A. (2007) ¿Hacia qué modelo de universidad converge Europa? Pedagogía Social. Revista Interuniversitaria, n. ${ }^{\circ} 14,53-74$.

Rodríguez BornaetXeA, F. (ed.) (2007) Psicología y conciencia. Barcelona, Kairós.

SÁEZ Alonso, R. (1991) El Conocimiento científico y el conocimiento mítico, asumidos en el método pedagógico. Revista Complutense de Educación, vol. 2, n. ${ }^{\circ}$ 2, 277-296.

Toulmin, S. (1990a) El descubrimiento del tiempo. Barcelona, Paidós.

- (1990b) Cosmopolis. The Hilen Agenda of Modernity. Chicago, University. 